\title{
The application of different appendicular skeletal muscle cutoff points and research definitions associated with health-related quality of life in Korean older people: data from KNHANES 2008-2011
}

Yeon-Pyo Kim ${ }^{1,2}$, Ju-Youn Joh¹, Sun Kim, Hwan-Sik Hwang ${ }^{1}$ and II-Seon Shin ${ }^{3^{*}}$

\begin{abstract}
Background: This study was conducted to determine the prevalence of a low appendicular skeletal muscle index (ASMI) using three cut-off points (mean ASMI-2SD of a gender-specific young reference group (aged 18-39 years), mean ASMI-1SD of a gender-specific young reference group, and the lower 20 percentile value of a gender-specific older group (aged $\geq 65$ years)) in Korean older people and the relationship between ASMI and subjective health-related quality of life.

Methods: This study utilized data acquired during the Korean National Health and Nutrition Examination Survey (KNHANES) from 2008-2011. Dual-energy X-ray absorptiometry body compositional data was obtained from a subsample of 6538 subjects (men 2804, women 3734) aged 18-39 and 4413 subjects (men 1872, women 2541) aged 65 years and older. The three definitions of low appendicular skeletal muscle and the EQ-5D-3 L-Korean descriptive system were applied to Korean older people.

Results: For the ASMI cutoff points used, in men, the three cutoff points were ASMI $2 S D\left(6.09 \mathrm{~kg} / \mathrm{m}^{2}\right)$, ASMI 20 $\left(6.48 \mathrm{~kg} / \mathrm{m}^{2}\right)$, and ASMI 1 SD $\left(6.95 \mathrm{~kg} / \mathrm{m}^{2}\right)$. In women, ASMI 2 SD $\left(4.38 \mathrm{~kg} / \mathrm{m}^{2}\right)$ was the lowest, followed by ASMI 1 SD $\left(4.96 \mathrm{~kg} / \mathrm{m}^{2}\right)$ and ASMI $20\left(5.33 \mathrm{~kg} / \mathrm{m}^{2}\right)$. Proportions of older subjects with a low ASMI using the three cutoff points were 9.7\% (ASMI 2SD) and 40.9\% (ASMI 1SD) in men, and 0.7\% (ASMI 2SD) and 7.4\% (ASMI 1SD) in women. By multivariate ordinal logistic regression analysis, men with a low ASMI had significantly high odd ratios for the three domains of mobility $(p<0.001)$, self-care $(p=0.005)$, and usual activities $(p=0.004)$ among the five domains of the EQ-5D and EQ-5D index $(p=0.010)$.

Conclusions: The ASMI 2SD cut-off points for older Koreans, $6.09 \mathrm{~kg} / \mathrm{m}^{2}$ for men and $4.38 \mathrm{~kg} / \mathrm{m}^{2}$ for women, resulted in low prevalences of a low ASM, that is, $9.7 \%$ for men and $0.7 \%$ for women, and showed low clinical usefulness due to very low determined prevalence in women. Hence, we suggest that the cut-off point of the lowest 20\% of Korean older people (men: $6.48 \mathrm{~kg} / \mathrm{m}^{2}$, women; $5.33 \mathrm{~kg} / \mathrm{m}^{2}$ ) be used for older Koreans.
\end{abstract}

Keywords: Sarcopenia, Korean, Older people

\footnotetext{
* Correspondence: isshin@chonnam.ac.kr

${ }^{3}$ Department of Psychiatry, Chonnam National University Hwasun Hospital,

322, Seoyang-ro, Hwasun-eup, Hwasun-gun, 519-809 Chonnam, Korea

Full list of author information is available at the end of the article
} 


\section{Background}

Life expectancies are increasing in most ethnic groups and countries, as are the survivals of older people with impaired physical functions and disabilities [1,2]. Accordingly, there is a great deal of research interest in precursors, including sarcopenia, in older people with impaired physical functions and disabilities [3,4].

Sarcopenia is a term applied by Irwin Rosenberg in 1989 to explain age-associated loss of muscle mass and muscle function [5]. Thereafter, researchers have added impaired functional performance and/or muscle weakness of clinical importance to the loss of muscle mass incorporated in the concept of sarcopenia. The Foundation for the National Institute of Health (FNIH) Sarcopenia Project defined sarcopenia as clinically relevant low muscle strength (weakness) and low lean mass in 2014 [6]. The Asian Working Group for Sarcopenia (AWGS) guideline also addressed cut-off points for Asian older people by modifying the European Working Group on Sarcopenia in Older People (EWGSOP) guideline [7].

The EWGSOP, International Working Group in Sarcopenia (IWGS) and AWGS guidelines define the sum of the muscle masses of the four limbs as appendicular skeletal mass (ASM) to calculate appendicular skeletal mass index (ASMI) as ASM/height ${ }^{2}\left(\mathrm{ASM} / \mathrm{m}^{2}\right)$ [3,4,8-10]. However, many other cutoff points have been proposed [11]. Previous studies among Asian older people showed substantially different cutoff points for low ASMI. Furthermore, the prevalences of low ASMI among Asian women were extremely low, which caused problems for researchers conducting studies on sarcopenia.

This study was conducted to determine the prevalence of low ASMI in accordance with three cutoff points in aged Korean ( $>65$ years), and to explore the relationship between low ASMI and subjective health-related quality of life.

\section{Methods \\ Subjects}

This study was based on data obtained from the 20082011 Korean National Health and Nutrition Examination Survey IV and V (KNHANES), a nationally-representative survey conducted by the Korean Ministry of Health and Welfare. KNHANES was a nationwide cross-sectional survey conducted in 1998-2012 by the Korean Center for Disease Control and Prevention (KCDC). KNHANES used stratified multi-stage clustered probability sampling to reflect the non-institutionalized Korean population. Participants completed a questionnaire that consisted of a health interview survey, a health behavior survey, a nutrition survey, and a health examination survey. A whole body dual energy X-ray absorptiometry (DXA) scan were performed on individuals $\geq 20$ years old from July 2008 to June 2009 and on individuals of $\geq 10$ years old from
July 2009 to May 2011 (excluding pregnant women), individuals with a height of $\geq 196 \mathrm{~cm}$ or a weight of $\geq 136 \mathrm{~kg}$ were excluded in accord with the KNHANES survey protocol. In addition, test results were treated as missing value in participants with implanted radio-opaque material (e.g., a prosthetic device, implant or other radio-opaque object) that could affect DXA results [12].

After excluding those without a whole body DXA scan (aged 18-39 years: 123 men and 214 women, aged 65-97 years: 105 men and 143 women), 6538 subjects (aged 18-39 years: 2,804 men, 3,743 women; the young reference group) and 4413 subjects (aged 65-97 years: 1872 men, 2541 women; the main analysis group) were included in the final analysis. Written informed consent was given by all participants and the protocol for KNHANES IV and V were approved by the Institutional Review Board of the KCDC (2008-04EXP-01-C, 2009-01CON-03-2C, 2010-02CON-21-C, 2011-02CON-06-C). Current study did not require additional Institutional Review Board approval because the KNHANES data set is publicly available.

\section{DXA measurements}

In the KNHANES study, whole body DXA examinations were conducted with a QDR4500A apparatus (Hologic, Bedford, MA, USA). All participants wore light clothing and removed all jewelry and other items that could interfere with DXA. The KNHANES data includes values for bone mineral content $(\mathrm{g})$, bone mineral density $\left(\mathrm{g} / \mathrm{cm}^{2}\right)$, fat mass (g), lean mass (including bone mineral content (g)), and fat percent (\%) for whole body and anatomical regions. From this data, ASM (kg) was calculated by summing the muscle masses of the four limbs, assuming that all non-fat and non-bone mass is skeletal muscle. ASMI was defined as ASM/height ${ }^{2}$ [13].

\section{Definition of low ASMI}

The following exclusion criteria were applied to the 6538 eligible subjects (aged 18 to 39 years), a prior diagnoses or treatment for diabetes, stroke, angina, myocardial infarction, thyroid disease, arthritis, asthma, chronic obstructive pulmonary disease, depression, chronic renal disease, chronic viral hepatitis, liver cirrhosis, or any malignancy. In addition, subjects hospitalized for any reason during the past year were also excluded $(n=1835)$. A total of 4703 (men 2096, women 2607) were recruited for the young (18-39 years) reference group. Low ASMI groups were defined using the following three cutoff point points: ASMI 2SD - the cutoff value of the lowest $2.28 \%$ in genderspecific young reference groups, ASMI 1SD - the cutoff value of the lowest $15.87 \%$ in gender-specific young reference groups, and ASMI 20 - the cutoff value of the lowest $20 \%$ in gender-specific older study participants.

Unlike previous studies, ASMI values of the young reference group in the current study were not normally 
distributed. Because young women's data showed high kurtosis, we used approximate values $(2.28 \%$, and $15.87 \%)$ in place of $1 \mathrm{SD}$ and $2 \mathrm{SD}$ values, respectively. These approximate values can be used in non-normally distributed data, whereas values of 1SD and 2SD can be used in normally distributed data [14].

\section{EQ-5D-3 L -Korean (EQ-5D)}

To measure health-related quality of life, we used the European Quality of Life-5 Dimensions (EQ-5D) instrument of the EuroQol group [15]. Validity of Korean version of EQ-5D (Spearman correlation coefficient with the first question of the Health Survey Short-Form 36: -0.51 in EQ-5D and -0.52 in EQ-VAS) and reliability (test-retest reliabilities using intra-class correlation: 0.75 in EQ-5D and -0.77 in EQ-VAS; agreement reliability using kappa statistics between 0.46 and 0.77 ) have already been demonstrated [16]. EQ-5D measures five single-item dimensions (mobility, self-care, usual activities, pain/discomfort, and anxiety/depression), each item has three levels of response (no, some, or extreme problems) (Figure 1). Responses to the five items are used to derive an overall health index score (using the weighting). In this study, we used the weighting suggestion of Nam et al. [17].

\section{Covariates}

The covariates included in the analysis were age, education, family income, body mass index (BMI), smoking

By placing a tick in one box in each group below, please indicate which statements best describe your own health state today.

Mobility

I have no problems in walking about

I have some problems in walking about

I am confined to bed

Self-Care

I have no problems with self-care

I have some problems washing or dressing myself

I am unable to wash or dress myself

Usual Activities (eg work, study, housework, family or leisure activities)

I have no problems with performing my usual activities

I have some problems with performing my usual activities

I am unable to perform my usual activities

Pain/Discomfort

I have no pain or discomfort

I have moderate pain or discomfort

I have extreme pain or discomfort

Anxiety/Depression

I am not anxious or depressed

I am moderately anxious or depressed

I am extremely anxious or depressed

To help people say how good or bad a health state is, we have drawn a scale (rather like a thermometer) on which the best state you

can imagine is marked 100 and the worst state you can imagine is marked 0 .

We would like you to indicate on this scale how good or bad your own health is today, in your opinion. Please do this by drawing a line from the box below to whichever point on the scale indicates how good or bad your health state is today.
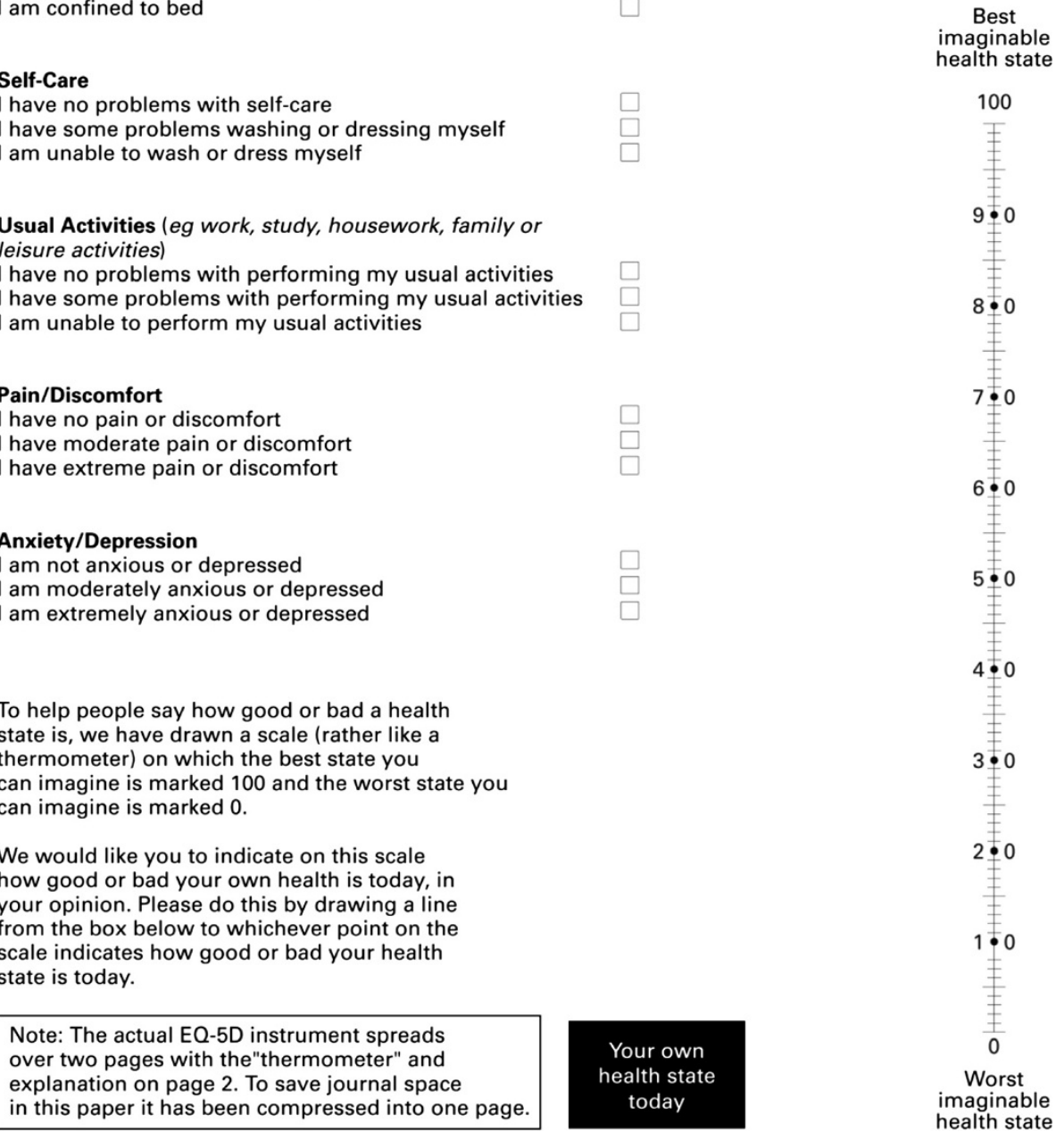

Figure 1 EQ-5D 3 (EQ-5D) questionnaire. Reproduced from [Journal of Neurology, Neurosurgery \& Psychiatry, Schrag. A. et al., 69(1), 67-73, 2000] with permission from EuroQol Group and BMJ Publishing Group Ltd. [18]. 
status, alcohol consumption, chronic illness, and number of chronic illness. BMI was used to assess obesity as determined by the World Health Organization Asia-Pacific criteria: underweight (BMI $<18.5 \mathrm{~kg} / \mathrm{m}^{2}$ ), normal weight (BMI: $18.5-22.9 \mathrm{~kg} / \mathrm{m}^{2}$ ), overweight (BMI: $23.0-27.4 \mathrm{~kg} / \mathrm{m}^{2}$ ), and obese (BMI $\geq 27.5 \mathrm{~kg} / \mathrm{m}^{2}$ ) [19]. Smoking status was categorized into four groups: never smokers (fewer than 100 cigarettes smoked during life), passive smokers (exposed to second-hand smoke for more than 1 hour a day in the home or workplace), current smokers, and former smokers. Alcohol consumption was assessed using the Korean version of the Alcohol Use Disorders Identification Test (AUDIT-K) and categorized based on scores into normal alcohol consumption group (men $<9$, women $<5$ ), harmful alcohol consumption group (men: 10-19, women: 6-9), and a suggestive alcohol dependent group (men $>20$, women $>10$ ) [20]. Chronic illnesses considered were cancer, diabetes, hypertension, stroke, coronary artery disease, arthritis, chronic pulmonary disease, depression, chronic renal failure, and chronic viral hepatitis/liver cirrhosis. These conditions were considered present when diagnosed by a physician at any time. We defined number of chronic illness as the sum of number of illness among the checklist of chronic illness.

\section{Statistical analyses}

Statistical analyses were conducted using the complex sample procedure because the KNHANES data set was selected using a representative, stratified, and clustered sampling method, not a random sampling method. The data in Table 1 were analyzed after weighting, because we needed sample distributions, such as, SDs and percentile values, for detailed ASMI analysis. Using these data, we determined low ASMI group depending on ASMI 1SD and ASMI 2SD and ASMI 20 value. Thereafter, univariate and multivariate analyses and ordinal regression analysis was performed using the five domains of EQ-5D and EQ-5D index as dependent variables and ASMI groups as independent variables. Because of a previously reported gender effect between ASMI and clinical outcomes, univariate and multivariate analyses were performed in each gender group [6]. In multivariate analysis, factors associated with ADL or IADL in previous studies, status of obesity using BMI, smoking, AUDIT-K, chronic illness, and numbers of chronic disease, were considered additional independent variables. Education level and family income, which are known to be significantly associated with EQ-5D, were also considered independent variables [17]. Here a backward elimination procedure was used. Accordingly, to this procedure, nonsignificant covariates ( $p>0.10$ ) were removed from the model until a final solution was reached. Statistical significance was accepted for $p$ values of $<0.05$. SPSS ver. 21.0 (SPSS, Chicago, IL, USA) was used for the statistical analysis.

\section{Results}

Mean and SD of ASMI in the young men group assuming a normal distribution was $7.81 \pm 0.91 \mathrm{~kg} / \mathrm{m}^{2}$ and in the young women group was $5.67 \pm 0.78 \mathrm{~kg} / \mathrm{m}^{2}$. If ASMI

Table 1 Anthropometric characteristics of the healthy young reference group and older study group by gender

\begin{tabular}{|c|c|c|c|c|}
\hline & \multicolumn{2}{|l|}{ Men } & \multicolumn{2}{|l|}{ Women } \\
\hline & Young reference group & $\geq 65$ years & Young reference group & $\geq 65$ years \\
\hline Sample size (no.) & 2096 & 1872 & 2607 & 2541 \\
\hline Weighted size (no.) & 6392302 & 2152083 & 5580819 & 3126111 \\
\hline Age (years) & $29(18-39)$ & $71(65-93)$ & $29(18-39)$ & $72(65-97)$ \\
\hline Height (cm) & $173.67 \pm 5.651$ & $164.81 \pm 5.745$ & $160.61 \pm 5.406$ & $150.47 \pm 5.764$ \\
\hline Weight (kg) & $71.39 \pm 11.241$ & $62.81 \pm 9.485$ & $56.04 \pm 9.520$ & $54.62 \pm 9.044$ \\
\hline Waist circumference (cm) & $81.29 \pm 9.250$ & $84.62 \pm 9.036$ & $72.44 \pm 9.011$ & $83.25 \pm 9.562$ \\
\hline Body mass index $\left(\mathrm{kg} / \mathrm{m}^{2}\right)$ & $23.65 \pm 3.415$ & $23.07 \pm 2.925$ & $21.72 \pm 3.495$ & $24.07 \pm 3.398$ \\
\hline Total fat mass (kg) & $15.54 \pm 6.248$ & $14.32 \pm 4.896$ & $17.81 \pm 5.782$ & $18.85 \pm 5.651$ \\
\hline Total body fat percentage (\%) & $21.40 \pm 6.020$ & $22.56 \pm 5.434$ & $31.47 \pm 5.585$ & $34.13 \pm 5.935$ \\
\hline ASM (kg) & $23.60 \pm 3.190$ & $19.44 \pm 2.756$ & $14.66 \pm 2.228$ & $13.36 \pm 1.905$ \\
\hline ASMI $\left(\mathrm{kg} / \mathrm{m}^{2}\right)$ & $7.81 \pm 0.909$ & $7.14 \pm 0.811$ & $5.67 \pm 0.777$ & $5.89 \pm 0.663$ \\
\hline ASMI 2SD cutoff point & 6.09 & & 4.38 & \\
\hline ASMI 1SD cutoff point & 6.95 & & 4.96 & \\
\hline ASMI 20 cutoff point & & 6.48 & & 5.33 \\
\hline
\end{tabular}

Weighted sample size reflects the number of objects and sample weights. Age is expressed as median (min-max). Other results are expressed as means \pm SDs. ASM = appendicular skeletal muscle mass. ASMI = appendicular skeletal muscle index. Low ASMI groups were defined as subjects whose ASMIs were lower than the following three cut-off points: ASMI 2SD - the value of the lowest $2.28 \%$ of the gender-specific young reference group, ASMI 1SD - the value of the lowest $15.87 \%$ of the gender-specific young reference group, and ASMI 20 - the value of lowest $20 \%$ of the gender-specific old age study group. 
in the young reference group had a normal distribution, the values of mean-2SD and the lowest $2.28 \%$ would have been the same. However, young men group (Kolmogorov-Smirnov $\mathrm{p}=0.020$ ) and the young women group (Kolmogorov-Smirnov $\mathrm{p}<0.001$ ) did not show a normal distribution due to high kurtosis. In the young men group, the mean-2SD value was $5.99 \mathrm{~kg} / \mathrm{m}^{2}$ and the lowest $2.28 \%$ value was $6.09 \mathrm{~kg} / \mathrm{m}^{2}$, and in the young women group corresponding values were $4.12 \mathrm{~kg} / \mathrm{m}^{2}$ and $4.38 \mathrm{~kg} / \mathrm{m}^{2}$, respectively. The difference between the two values was higher in the young women group $\left(0.26 \mathrm{~kg} / \mathrm{m}^{2}\right.$ vs. 0.10 $\left.\mathrm{kg} / \mathrm{m}^{2}\right)$. The ASMI cutoff points in older men were ASMI 2SD $\left(6.09 \mathrm{~kg} / \mathrm{m}^{2}\right)$, ASMI $20\left(6.48 \mathrm{~kg} / \mathrm{m}^{2}\right)$, and ASMI 1SD $\left(6.95 \mathrm{~kg} / \mathrm{m}^{2}\right)$, and corresponding values for older women were ASMI 2SD $\left(4.38 \mathrm{~kg} / \mathrm{m}^{2}\right)$, ASMI 1 SD $\left(4.96 \mathrm{~kg} / \mathrm{m}^{2}\right)$, and ASMI $20\left(5.33 \mathrm{~kg} / \mathrm{m}^{2}\right)$ (Table 1). Characteristics of the older study participants are summarized in Table 2 . The proportions in low group for each cutoff point for older men study subjects were 9.7\% (ASMI 2SD) and 40.9\% (ASMI 1SD), respectively, and for older women were $0.7 \%$ (ASMI 2SD) and 7.4\% (ASMI 1SD), respectively (Table 3). By univariate analysis with ordinal regression analysis using the complex sample procedure, low ASMI had significantly associated with poorer outcome of five domains of EQ-5D and of the EQ-5D index in older men. More specifically, ASMI ranging from ASMI 2SD to ASMI 20 had higher cumulative odds ratios (ORs) than those with those a normal ASMI [mobility 2.28 (95\% Confidence Interval (CI): 1.55-3.35), self-care (OR 2.10, CI: 1.10-4.00), usual activities (OR 2.40, CI: 1.53-3.77), pain/discomfort (OR 1.63, CI:1.13-2.36), anxiety/depression (OR 1.76, CI:1.01-3.09), and EQ-5D index (OR 2.09, CI: 1.45-3.02)] in older men (Table 4). Low ASMI had significantly associated with poorer outcome of domains of self-care and anxiety/depression among the five domains of EQ-5D in older women. More specifically, ASMIs ranging from ASMI 2SD to ASMI 20 had a higher OR for difficulty in self care (OR 1.20, CI: 0.82-1.74) and prevalence of anxiety/depression (OR 0.78, CI: 0.54-1.12) in older women (Table 5).

By multivariate ordinal logistic regression, low ASMI had significantly higher ORs for the three domains of mobility, self-care, and usual activities among the five domains of EQ-5D in older men (Table 4). In detail, the findings with ASMI 20 were mobility (OR 2.54, CI: 1.63-3.95), usual activities (OR 2.31, CI: 1.31-4.09), and EQ-5D index (OR 1.86, CI: 1.23-2.82) in older men (Table 6). However these correlations were not significant for all three cutoff points ASMI 2SD, ASMI 1SD (except ASMI 2SD), and ASMI 20 (except ASMI 1SD) in older women (Table 7).

\section{Discussion}

In this Korean national representative sample, the prevalences of low ASMI according to a cutoff point of ASMI
2SD were $9.7 \%$ and $0.7 \%$ for older ( $\geq 65$ years) men and women, respectively, however, according to cutoff point of ASMI 1SD, $40.9 \%$ and $7.4 \%$ of the older men and women, respectively, had a low ASMI. In addition, only older men with a low ASMI were found to have a low health-related quality of life.

The means and SDs of ASMI were $7.14 \pm 0.81 \mathrm{~kg} / \mathrm{m}^{2}$ and $5.89 \pm 0.66 \mathrm{~kg} / \mathrm{m}^{2}$ in older Korean men and women, respectively, which are a little lower than those of previous studies. The New Mexico Elder Health Survey for Hispanic and non-Hispanic Americans conducted from May 1993 to September 1995 reported a mean ASMI was $7.7 \pm 0.7 \mathrm{~kg} / \mathrm{m}^{2}$ in older men and $5.9 \pm 0.7 \mathrm{~kg} / \mathrm{m}^{2}$ in older women [13]. The Health ABC study on Americans conducted from 1997 to 1998 reported a mean ASMI in European Americans of $7.8 \pm 0.9 \mathrm{~kg} / \mathrm{m}^{2}$ in older men and of $6.1 \pm 0.8 \mathrm{~kg} / \mathrm{m}^{2}$ in older women [21]. A Japanese study conducted from July 2002 to June 2003 reported mean ASMIs of $7.61 \pm 0.7 \mathrm{~kg} / \mathrm{m}^{2}$ in older men and $6.32 \pm 0.6 \mathrm{~kg} / \mathrm{m}^{2}$ in older women [22]. Finally, a study in Taiwan published in 2013 reported mean ASMIs of $7.6 \pm 0.8 \mathrm{~kg} / \mathrm{m}^{2}$ and $6.4 \pm$ $0.6 \mathrm{~kg} / \mathrm{m}^{2}$ in older men and women, respectively [23].

On the other hand, mean ASMIs in the young reference group in the present study were $7.81 \pm 0.91 \mathrm{~kg} / \mathrm{m}^{2}$ and $5.67 \pm 0.78 \mathrm{~kg} / \mathrm{m}^{2}$ in men and women, which are markedly lower than the values obtained in the American study, which reported mean ASMIs for a young reference group (18-40 years) of $8.6 \pm 1.1 \mathrm{~kg} / \mathrm{m}^{2}$ and $7.3 \pm 0.9 \mathrm{~kg} / \mathrm{m}^{2}$ in men and women [13]. The present findings are also lower than those in the Taiwanese study, which reported mean ASMIs in a disease-free young reference group (20-40 years) of $8.2 \pm 0.9 \mathrm{~kg} / \mathrm{m}^{2}$ and $5.9 \pm 0.8 \mathrm{~kg} / \mathrm{m}^{2}$ in men and women [23].

In The New Mexico Elder Health Survey for Hispanic and non-Hispanic Americans, the mean ASMIs of older men and women were approximately at the 87 and 80 percentiles, respectively, of the young reference group [13]. In our study, the mean ASMIs of older men and women were at the 91 and 104 percentiles, of the young reference group, and the mean ASMI of older women was higher than the mean ASMI of the young woman reference group.

The proportion of older people with an ASMI two standard deviations below the mean ASMI of the young reference group also displayed a marked difference in the present study as compared with previously published values. In our study, older people with below ASMI 2SD were 9.7\% in older men and $0.7 \%$ in older women, as compared to $13 \%$ at 61 to 70 years of age, $24 \%$ at 71 to 80 years of age, and $50 \%$ at aged 81 aged and older [13], and $28 \%$ in men $\geq 70$ years of age and $52 \%$ in women $\geq 70$ years of age [24].

Interestingly, many studies on sarcopenia in Asian populations have not used the standard ASMI cutoff of two standard deviations below the mean ASMI of the young reference group. The ASMI 1SD cutoff points 
Table 2 General characteristics of older study participants by gender

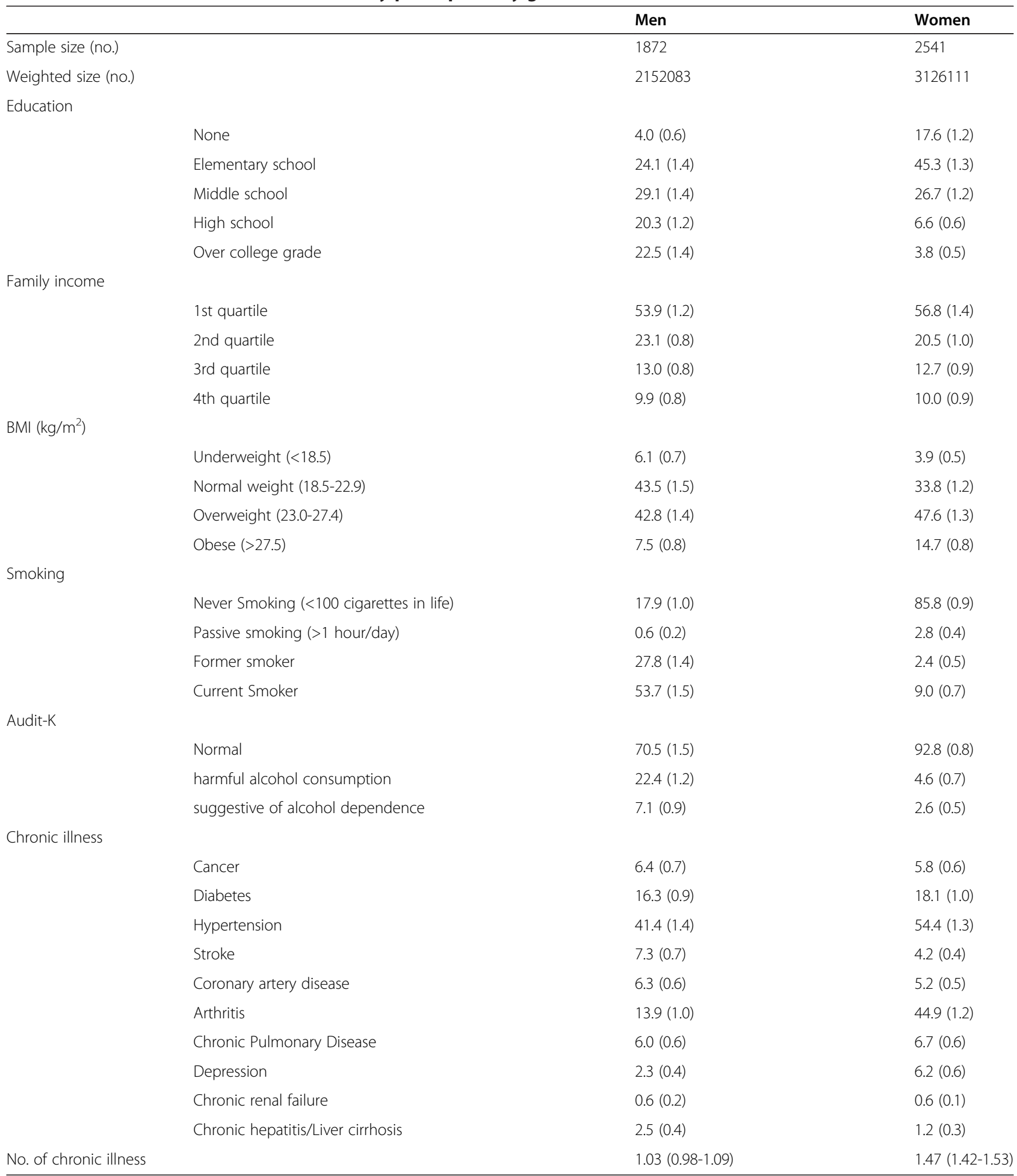

Weighted sample size reflects the number of objects and the sample weights. Results are expressed as percentages (standard errors). Numbers of chronic illnesses are expressed as means (95\% Confidence Index).

used in Japanese and Korean studies were $6.53 \mathrm{~kg} / \mathrm{m}^{2}$ in men and $5.21 \mathrm{~kg} / \mathrm{m}^{2}$ in women and $7.09 \mathrm{~kg} / \mathrm{m}^{2}$ in men and $5.27 \mathrm{~kg} / \mathrm{m}^{2}$ in women, while a study in Taiwan used the lower 20 percentile values of gender specific healthy young age individuals $[22,23,25]$. Considering the diagnostic criteria of sarcopenia recommended by the IWGS, EWGSOP, and AWGS, these sets of research standards are atypical. It is possible that the use of the ASMI 2SD 
Table 3 Prevalence of low ASMI and EQ-5D for older study participants by gender

\begin{tabular}{lll}
\hline & Men & Women \\
\hline Sample size (no.) & 1872 & 2541 \\
Weighted size (no.) & 2152083 & 3126111 \\
ASMI & & \\
$\quad$ ASMI 2SD & $9.7(0.9)$ & $0.7(0.2)$ \\
ASMI 1SD & $40.9(1.5)$ & $7.4(0.7)$ \\
$\quad$ ASMI 20 & $20.0(1.2)$ & $20.0(1.1)$ \\
EQ5D & & \\
$\quad$ Mobility & &
\end{tabular}

$\begin{array}{cll}\text { No problem } & 65.4(1.4) & 42.8(1.3) \\ \text { Some problems } & 32.9(1.3) & 53.4(1.3) \\ \text { Extreme problems } & 1.7(0.4) & 3.8(0.5) \\ \text { Self-Care } & & \\ \text { No problem } & 90.4(0.9) & 80.9(1.0) \\ \text { Some problems } & 8.6(0.8) & 17.4(1.0) \\ \text { Extreme problems } & 1.0(0.2) & 1.7(0.3)\end{array}$

Usual Activities (e.g., work, study, housework, family or leisure activity)

$\begin{array}{lll}\text { No problem } & 76.6(1.3) & 61.9(1.2) \\ \text { Some problems } & 19.9(1.2) & 31.5(1.1) \\ \text { Extreme problems } & 3.5(0.5) & 6.6(0.7)\end{array}$

Pain/Discomfort

$\begin{array}{lll}\text { No problem } & 67.3(1.3) & 47.3(1.3) \\ \text { Some problems } & 26.9(1.2) & 38.2(1.2) \\ \text { Extreme problems } & 5.8(0.6) & 14.5(0.8)\end{array}$

Anxiety/Depression

$\begin{array}{lll}\text { No problem } & 88.9(0.9) & 79.5(1.0) \\ \text { Some problems } & 9.3(0.7) & 17.4(0.9) \\ \text { Extreme problems } & 1.9(0.5) & 3.2(0.4)\end{array}$

EQ5D index

$\begin{array}{lll}1 & 52.6(1.4) & 28.9(1.1) \\ 0.775-0.999 & 26.8(1.3) & 33.5(1.3) \\ <0.774 & 20.6(1.2) & 37.7(1.3)\end{array}$

The weighted sample size reflects the number of objects and the sample weights. Results are expressed as percentages (standard errors). Low ASMI groups were defined as subjects whose ASMIs were lower than the following three cut-off points: ASMI 2SD - the value of the lowest $2.28 \%$ of the gender-specific young reference group, ASMI 1SD - the value of the lowest $15.87 \%$ of the gender-specific young reference group, and ASMI 20 - the value of lowest $20 \%$ of the gender-specific old age study group.

value as a cutoff point in the aforementioned studies would have resulted in an unacceptably small proportion of sarcopenic older individuals. Other studies in Asians may have been similarly hampered.

A number of reasons can be suggested for the low proportions of older people with an ASMI 2SD below the mean ASMI of young reference groups in the present study. First, the body types of Koreans are changing due to improvements in living standards. According to the
2010 Size-Korea survey of body measurement, the average height of Koreans increased steadily up to 2003 and remained constant thereafter, whereas the body types of Koreans have consistently changed. In that survey, the proportion of body shape of Korean 20s women changed to 7.3-head units tall as compared with 20 years earlier a 7.2-head units tall, and leg lengths of Korean women were nearly 2 centimeters longer than 30 years earlier [26]. Second, Korean women in their 20s and 30s can have poor weight reducing habits, and rely diet restriction rather than exercise. This reflects a distorted body image, with a desire to have a body type similar to Caucasian women, Korean women tend to prefer a slim body with focus on diet restriction, have a negative effect on muscle development [27-29]. As a result, muscle mass in Korean women has been consistently reported to be decreasing more so than body fat because of repetitive low calorie diets and accompanying cycles of weight loss and weight gain $[27,30,31]$. These results are consistent with the results of the Size-Korea survey, which found that mean BMI of subjects in their late 20s significantly decreased over time and the proportion of underweight individuals (as determined by BMI) increased to $15.7 \%$ in 2010 from $11.5 \%$ in 2003, while decreases in waist circumference was not significant over the same period [26]. As a result, in the present study, the mean ASMI of the young reference women group was lower than the mean ASMI of the older women group ( $\geq 65$ years of age) and the proportion of older women in the below ASMI 2SD group was excessively low.

Because ASMI values of the young reference group in the current study did not show a normal distribution, we used alternative values that corresponded to lowest $2.28 \%$ and $15.87 \%$ values in the young reference group in place of $1 \mathrm{SD}$ and $2 \mathrm{SD}$ values, respectively. After making this adjustment, the cutoff point of ASMI 2SD increased from $4.12 \mathrm{~kg} / \mathrm{m}^{2}$ to $4.38 \mathrm{~kg} / \mathrm{m}^{2}$ in older women, and had we applied the real ASMI 2SD value $\left(4.12 \mathrm{~kg} / \mathrm{m}^{2}\right)$ only four older women would have had a low ASMI.

It is suggested that the existing sarcopenia diagnostic criteria using ASMI of a young reference group of the same gender and race is inadequate for Koreans. Considering clinical outcomes later in life, re-establishment of optimal ASMI cut-off points is needed.

We performed multivariate ordinal logistic regression analysis to examine the association between the low ASMI group based on the three established ASMI cutoff points mentioned and EQ-5D determined health-related quality of life. Low ASMI had significantly associated with poorer outcome of three domains of mobility, selfcare, and usual activities among the five domains of the EQ-5D in older men. However, in older women with a low ASMI, these correlations were not significant for any of the three ASMI cutoff points. These findings are 
Table 4 Univariate analysis of five domains of EQ-5D and EQ5D index according to ASMI in older men

\begin{tabular}{|c|c|c|c|c|c|c|}
\hline & & Estimates & Standard error & Cumulative odds ratio & $95 \% \mathrm{Cl}$ & $\mathrm{p}$ value \\
\hline \multirow[t]{5}{*}{ Mobility } & & & & & & $<0.001^{*}$ \\
\hline & ASMI 2SD & 0.818 & 0.192 & 2.267 & $1.554-3.308$ & $<0.001$ \\
\hline & ASMI 20 & 0.824 & 0.195 & 2.280 & $1.554-3.346$ & $<0.001$ \\
\hline & ASMI 1SD & 0.311 & 0.159 & 1.365 & $0.998-1.867$ & 0.051 \\
\hline & Normal & Reference & & & & \\
\hline \multirow[t]{5}{*}{ Self-care } & & & & & & $<0.001^{*}$ \\
\hline & ASMI 2SD & 1.152 & 0.250 & 3.165 & $1.938-5.169$ & $<0.001$ \\
\hline & ASMI 20 & 0.743 & 0.328 & 2.103 & $1.104-4.007$ & 0.024 \\
\hline & ASMI 1SD & 0.252 & 0.294 & 1.286 & $0.721-2.294$ & 0.393 \\
\hline & Normal & Reference & & & & \\
\hline \multicolumn{3}{|c|}{ Usual activities } & & & & $<0.001^{*}$ \\
\hline & ASMI 2SD & 1.153 & 0.195 & 3.169 & $2.159-4.651$ & $<0.001$ \\
\hline & ASMI 20 & 0.875 & 0.230 & 2.398 & $1.526-3.767$ & $<0.001$ \\
\hline & ASMI 1SD & 0.500 & 0.181 & 1.648 & $1.156-2.350$ & $<0.001$ \\
\hline & Normal & Reference & & & & \\
\hline \multicolumn{3}{|c|}{ Pain/discomfort } & & & & $0.010^{*}$ \\
\hline & ASMI 2SD & 0.576 & 0.196 & 1.778 & $1.209-2.615$ & 0.004 \\
\hline & ASMI 20 & 0.490 & 0.188 & 1.632 & $1.128-2.362$ & 0.009 \\
\hline & ASMI 1SD & 0.192 & 0.148 & 1.212 & $0.906-1.621$ & 0.194 \\
\hline & Normal & Reference & & & & \\
\hline \multicolumn{3}{|c|}{ Anxiety/depression } & & & & $0.012^{*}$ \\
\hline & ASMI 2SD & 0.788 & 0.272 & 2.199 & $1.289-3.752$ & 0.004 \\
\hline & ASMI 20 & 0.567 & 0.286 & 1.763 & $1.006-3.090$ & 0.048 \\
\hline & ASMI 1SD & 0.338 & 0.227 & 1.402 & $0.899-2.188$ & 0.136 \\
\hline & Normal & Reference & & & & \\
\hline \multirow[t]{5}{*}{ EQ5D inde } & & & & & & $<0.001^{*}$ \\
\hline & ASMI 2SD & 0.879 & 0.161 & 2.408 & $1.755-3.304$ & $<0.001$ \\
\hline & ASMI 20 & 0.736 & 0.187 & 2.088 & $1.445-3.016$ & $<0.001$ \\
\hline & ASMI 1SD & 0.305 & 0.142 & 1.357 & $1.028-1.792$ & 0.031 \\
\hline & Normal & reference & & & & \\
\hline
\end{tabular}

The weighted sample size reflects the number of objects and the sample weights. $95 \% \mathrm{Cl}$ : $95 \%$.

Confidential Index for Cumulative Odds Ratio. *Adjustment for multiple comparisons was performed using the Sequential Sidak method. Low ASMI groups were defined as subjects whose ASMls were lower than the following three cutoff points: ASMI 2 SD - the value of the lowest $2.28 \%$ of the gender-specific young reference group, ASMI 1SD - the value of the lowest $15.87 \%$ of the gender-specific young reference group, and ASMI 20 - the value of lowest $20 \%$ of the gender-specific old age study group.

consistent with previous finding of a stronger correlation between sarcopenia and functional decline and disability in older men [4]. Additional studies about genderassociated differences in muscle aging are needed.

Although the association between the low ASMI group based on ASMI 20 and any domain of EQ-5D was not significant in older women, the ASMI 20 cutoff point was significantly associated with EQ-5D in older men. On the other hand, the ASMI 2SD and ASMI 1SD cutoff points may not be adequate to diagnose sarcopenia in older Koreans because the proportions of older people with sarcopenia are quite variable. Studies are needed on the optimal ASMI cutoff point with respect to muscle function and clinical outcome for Koreans. We suggest that an ASMI 20 cutoff point (men $6.48 \mathrm{~kg} / \mathrm{m}^{2}$, women $5.33 \mathrm{~kg} / \mathrm{m}^{2}$ ) is appropriate for older people for the assessment of low ASM, until an optimal cutoff point is determined. Further studies on country/population specific ASMI cutoff point are needed in different communities.

This study had several strengths. First, our data were collected from a nationwide survey that reflected the community-dwelling Korean population. In particular, because the young reference group was large (2096 men 
Table 5 Univariate analysis of five domains of EQ-5D and EQ5D index according to ASMI in older women

\begin{tabular}{|c|c|c|c|c|c|c|}
\hline & & Estimates & Standard error & Cumulative odds ratio & $95 \% \mathrm{Cl}$ & $p$ value \\
\hline \multirow[t]{5}{*}{ Mobility } & & & & & & $0.264^{*}$ \\
\hline & ASMI 2SD & 0.046 & 0.585 & 1.047 & $0.332-3.303$ & 0.937 \\
\hline & ASMI 1SD & -0.102 & 0.227 & 0.903 & $0.579-1.410$ & 0.654 \\
\hline & ASMI 20 & -0.242 & 0.146 & 0.785 & $0.589-1.045$ & 0.097 \\
\hline & Normal & Reference & & & & \\
\hline \multirow[t]{5}{*}{ Self-care } & & & & & & $0.011^{*}$ \\
\hline & ASMI 2SD & 1.634 & 0.561 & 5.122 & $1.702-15.418$ & 0.004 \\
\hline & ASMI 1SD & 0.412 & 0.252 & 1.510 & $0.921-2.477$ & 0.102 \\
\hline & ASMI 20 & 0.179 & 0.190 & 1.196 & $0.824-1.737$ & 0.345 \\
\hline & Normal & Reference & & & & \\
\hline \multicolumn{3}{|c|}{ Usual activities } & & & & $0.087^{*}$ \\
\hline & ASMI 2SD & 1.238 & 0.569 & 3.450 & $1.129-10.542$ & 0.030 \\
\hline & ASMI 1SD & 0.188 & 0.220 & 1.206 & $0.784-1.857$ & 0.394 \\
\hline & ASMI 20 & 0.231 & 0.157 & 1.260 & $0.924-1.716$ & 0.143 \\
\hline & Normal & Reference & & & & \\
\hline \multicolumn{3}{|c|}{ Pain/discomfort } & & & & $0.522^{*}$ \\
\hline & ASMI 2SD & 1.101 & 0.894 & 3.009 & $0.520-17.416$ & 0.218 \\
\hline & ASMI 1SD & 0.057 & 0.191 & 1.058 & $0.728-1.539$ & 0.767 \\
\hline & ASMI 20 & -0.080 & 0.144 & 0.923 & $0.695-1.225$ & 0.578 \\
\hline & Normal & Reference & & & & \\
\hline \multicolumn{3}{|c|}{ Anxiety/depression } & & & & $0.021^{*}$ \\
\hline & ASMI 2SD & 1.498 & 0.555 & 4.471 & $1.504-13.290$ & 0.007 \\
\hline & ASMI 1SD & 0.441 & 0.261 & 1.554 & $0.931-2.596$ & 0.092 \\
\hline & ASMI 20 & -0.253 & 0.188 & 0.776 & $0.536-1.123$ & 0.179 \\
\hline & Normal & Reference & & & & \\
\hline \multirow[t]{5}{*}{ EQ5D inde } & & & & & & $0.858^{*}$ \\
\hline & ASMI 2SD & 0.637 & 0.898 & 1.890 & $0.324-11.034$ & 0.479 \\
\hline & ASMI 1SD & 0.087 & 0.211 & 1.091 & $0.721-1.650$ & 0.681 \\
\hline & ASMI 20 & -0.006 & 0.152 & 0.994 & $0.738-1.340$ & 0.969 \\
\hline & Normal & Reference & & & & \\
\hline
\end{tabular}

The weighted sample size reflects the number of objects and the sample weights. $95 \% \mathrm{Cl}: 95 \%$.

Confidential Index for Cumulative Odds Ratio. *Adjustment for multiple comparisons was performed using the Sequential Sidak method. Low ASMI groups were defined as subjects whose ASMIs were lower than the following three cutoff points: ASMI 2 SD - the value of the lowest $2.28 \%$ of the gender-specific young reference group, ASMI 1SD - the value of the lowest $15.87 \%$ of the gender-specific young reference group, and ASMI 20 - the value of lowest $20 \%$ of the gender-specific old age study group.

and 2607 women) and was nationally representative, the ASMI distribution based on the young reference group can be considered reliable. Second, we performed analyses in a gender-specific manner. We found that the association between height-adjusted ASM and EQ-5D differed in older men and women, which is consistent with the findings of the recent FNIH study, in which height-adjusted ASM was found to be more useful for men and weight-adjusted ASM for women with respect skeletal muscle functions [6], implying a possible underlying gender effect.
Finally, to our best knowledge, this study is the first report of a significant association between low ASMI and healthrelated quality of life. Our finding implies a possible relationship between sarcopenia and health-related quality of life, however, further studies are required to confirm the relation.

On the other hand, the present study has following limitations that warrant consideration. First, the study lacked investigation of cause-effect relationships because of its cross-sectional nature. Future prospective cohort studies are needed for such investigations. Second, previous studies analyzed ADL and IADL as dependent variables, 
Table 6 Multivariate analysis of five domains of EQ-5D and EQ5D index according to ASMI in older men

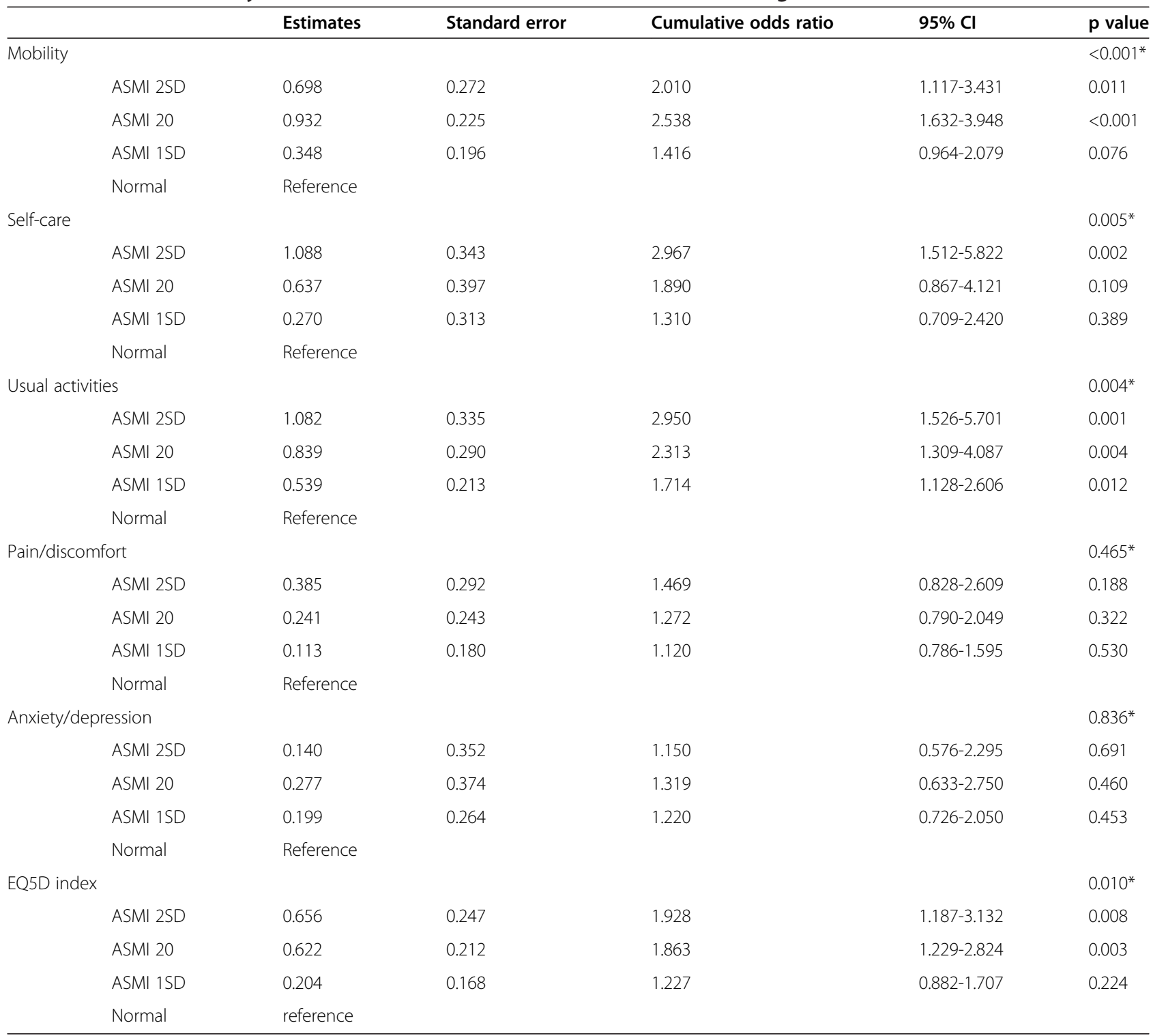

The weighted sample size reflects the number of objects and the sample weights. $95 \% \mathrm{Cl}: 95 \%$.

Confidential Index for Cumulative Odds Ratio. *Adjustment for multiple comparisons was performed using the Sequential Sidak method for obesity criteria, family income, education, smoking, alcohol consumption criteria (AUDIT-K), chronic illness (cancer, diabetes, hypertension, stroke, coronary artery disease, arthritis, chronic pulmonary disease, depression, chronic renal failure, chronic viral hepatitis/ liver cirrhosis), and number of chronic diseases. Low ASMI groups were defined as subjects whose ASMIs were lower than the following three cutoff points: ASMI 2SD - the value of the lowest $2.28 \%$ of the gender-specific young reference group, ASMI 1 SD - the value of the lowest $15.87 \%$ of the gender-specific young reference group, and ASMI 20 - the value of lowest $20 \%$ of the gender-specific old age study group.

whereas we used EQ-5D as a dependent variable. Although EQ-5D contains items similar to ADL and IADL, such as, mobility, self-care, and usual activities, direct comparisons might be inappropriate. Third, we did not consider muscle strength and muscle function and we used only height-adjusted ASM. The recent guidelines (EWGSOP, IWGS, AWGS and FNIH guideline) recommend to diagnose sarcopenia as reduced muscle mass related to muscle strength and function such as grip strength and gait speed, not only reduced muscle mass itself. However, our KNHANES data set did not contain information on muscle strength and muscle function, thus we have analysed only muscle mass in this study. Further studies are needed using our suggested ASM cutoff point and the FNIH guidelines, which represent the state of the art, recommend BMI-adjusted ASM. Finally, we did not include physical activity as a potential confounder because KNHANES IV and KNHANES $\mathrm{V}$ had different items of measurement. Further studies on the topic are needed. 
Table 7 Multivariate analysis of five domains of EQ-5D and EQ5D index according to ASMI in older women

\begin{tabular}{|c|c|c|c|c|c|c|}
\hline & & Estimates & Standard error & Cumulative odds ratio & $95 \% \mathrm{Cl}$ & $p$ value \\
\hline \multirow[t]{5}{*}{ Mobility } & & & & & & $0.093^{*}$ \\
\hline & ASMI 2SD & -1.407 & 0.826 & 0.245 & $0.048-1.240$ & 0.089 \\
\hline & ASMI 1SD & 0.157 & 0.299 & 1.170 & $0.650-2.106$ & 0.599 \\
\hline & ASMI 20 & -0.469 & 0.218 & 0.626 & $0.408-0.961$ & 0.032 \\
\hline & Normal & & & & & \\
\hline \multirow[t]{5}{*}{ Self-care } & & & & & & $0.974^{*}$ \\
\hline & ASMI 2SD & -0.227 & 1.265 & 0.797 & $0.066-9.561$ & 0.858 \\
\hline & ASMI 1SD & 0.136 & 0.360 & 1.146 & $0.565-2.325$ & 0.705 \\
\hline & ASMI 20 & -0.042 & 0.288 & 0.959 & $0.544-1.689$ & 0.884 \\
\hline & Normal & & & & & \\
\hline \multicolumn{2}{|c|}{ Usual activities } & & & & & $0.710^{*}$ \\
\hline & ASMI 2SD & -0.111 & 1.354 & 0.895 & $0.063-12.791$ & 0.935 \\
\hline & ASMI 1SD & 0.315 & 0.328 & 1.370 & $0.719-2.610$ & 0.338 \\
\hline & ASMI 20 & -0.014 & 0.228 & 0.986 & $0.630-1.544$ & 0.951 \\
\hline & Normal & & & & & \\
\hline \multicolumn{2}{|c|}{ Pain/discomfort } & & & & & $0.299^{*}$ \\
\hline & ASMI 2SD & -1.274 & 1.375 & 0.280 & $0.019-4.165$ & 0.354 \\
\hline & ASMI 1SD & -0.082 & 0.287 & 0.921 & $0.524-1.619$ & 0.775 \\
\hline & ASMI 20 & -0.306 & 0.192 & 0.737 & $0.505-1.074$ & 0.112 \\
\hline & Normal & & & & & \\
\hline \multicolumn{2}{|c|}{ Anxiety/depression } & & & & & $0.754^{*}$ \\
\hline & ASMI 2SD & -0.754 & 1.210 & 0.470 & $0.044-5.070$ & 0.534 \\
\hline & ASMI 1SD & 0.177 & 0.309 & 1.194 & $0.651-2.190$ & 0.566 \\
\hline & ASMI 20 & -0.248 & 0.279 & 0.780 & $0.451-1.349$ & 0.374 \\
\hline & Normal & & & & & \\
\hline \multirow[t]{5}{*}{ EQ5D inde } & & & & & & $0.490^{*}$ \\
\hline & ASMI 2SD & -1.828 & 1.427 & 0.161 & $0.010-2.652$ & 0.201 \\
\hline & ASMI 1SD & 0.113 & 0.317 & 1.120 & $0.601-2.085$ & 0.721 \\
\hline & ASMI 20 & -0.182 & 0.234 & 0.833 & $0.527-1.319$ & 0.436 \\
\hline & Normal & & & & & \\
\hline
\end{tabular}

The weighted sample size reflects the number of objects and the sample weights. $95 \% \mathrm{Cl}$ : $95 \%$.

Confidential Index for Cumulative Odds Ratio. *Adjustment for multiple comparisons was performed using the Sequential Sidak method for obesity criteria, family income, education, smoking, alcohol consumption criteria (AUDIT-K), chronic illness (cancer, diabetes, hypertension, stroke, coronary artery disease, arthritis, chronic pulmonary disease, depression, chronic renal failure, chronic viral hepatitis/ liver cirrhosis), and Number of chronic disease. Low ASMI groups were defined as subjects whose ASMIs were lower than the following three cutoff points: ASMI 2 SD - the value of the lowest $2.28 \%$ of the gender-specific young reference group, ASMI 1SD - the value of the lowest $15.87 \%$ of the gender-specific young reference group, and ASMI 20 - the value of lowest $20 \%$ of the gender-specific old age study group.

\section{Conclusions}

Among Korean older people, the prevalence of low ASM was $9.7 \%$ in men and only $0.7 \%$ in women when an ASMI 2SD cutoff point of $6.09 \mathrm{~kg} / \mathrm{m}^{2}$ was used for men and $4.38 \mathrm{~kg} / \mathrm{m}^{2}$ for women, which lowers its clinical usefulness because of the extremely low determined prevalence in women. Hence, we suggest that the cutoff point of the lowest $20 \%$ of Korean older people (men: $6.48 \mathrm{~kg} / \mathrm{m}^{2}$, women; $5.33 \mathrm{~kg} / \mathrm{m}^{2}$ ) be used for older Korean people.
Competing interests

The authors declare that they have no competing interests.

\section{Authors' contributions}

YPK, HSH and ILS conceptualized the study design. YPK conducted analyses. YPK, JYJ and SK wrote the first draft of the paper. All authors critically revised the manuscript and read and approved the final version.

\section{Author details}

${ }^{1}$ Department of Family Medicine, Chonnam National University Hwasun Hospital, 322, Seoyang-ro, Hwasun-eup, Hwasun-gun, ChonnamKorea 
519-809; HwasunKorea. ${ }^{2}$ Center for Aging and Geriatrics, Chonnam National University Medical School, Gwang-Ju, Korea. ${ }^{3}$ Department of Psychiatry, Chonnam National University Hwasun Hospital, 322, Seoyang-ro, Hwasun-eup, Hwasun-gun, 519-809 Chonnam, Korea.

Received: 22 May 2014 Accepted: 17 December 2014 Published: 23 December 2014

\section{References}

1. Freedman VA, Martin LG, Schoeni RF: Recent trends in disability and functioning among older adults in the United States: a systematic review. JAMA 2002, 288(24):3137-3146.

2. Manton KG, Lamb VL, Gu X: Medicare cost effects of recent U.S. disability trends in the elderly: future implications. J Aging Health 2007, 19(3):359-381.

3. Cruz-Jentoft AJ, Baeyens JP, Bauer JM, Boirie Y, Cederholm T, Landi F, Martin FC, Michel JP, Rolland Y, Schneider SM, Topinkova E, Vandewoude M, Zamboni M: Sarcopenia: European consensus on definition and diagnosis: report of the European working group on sarcopenia in older people. Age Ageing 2010, 39(4):412-423.

4. Fielding RA, Vellas B, Evans WJ, Bhasin S, Morley JE, Newman AB, Abellan Van Kan G, Andrieu S, Bauer J, Breuille D, Cederholm T, Chandler J, Meynard CD, Donini L, Harris T, Kannt A, Guibert FK, Onder G, Papanicolaou D, Rolland Y, Rooks D, Sieber C, Souhami E, Verlaan S, Zamboni M: Sarcopenia: an undiagnosed condition in older adults. Current consensus definition: prevalence, etiology, and consequences. International working group on sarcopenia. J Am Med Assoc 2011, 12(4):249-256.

5. Rosenberg IH: Summary comments. Am J Clin Nutr 1989, 50(5):1231-1233.

6. Studenski SA, Peters KW, Alley DE, Cawthon PM, McLean RR, Harris TB, Ferrucci L, Guralnik JM, Fragala MS, Kenny AM: The FNIH sarcopenia project: rationale, study description, conference recommendations, and final estimates. J Gerontol A: Biol Med Sci 2014, 69(5):547-558,

7. Chen L-K, Liu L-K, Woo J, Assantachai P, Auyeung T-W, Bahyah KS, Chou M-Y, Chen L-Y, Hsu P-S, Krairit O: Sarcopenia in Asia: consensus report of the Asian working group for sarcopenia. J Am Med Assoc 2014, 15(2):95-101.

8. Prado CM, Wells JC, Smith SR, Stephan BC, Siervo M: Sarcopenic obesity: a critical appraisal of the current evidence. Clin Nutr 2012, 31(5):583-601.

9. Clark BC, Manini TM: Functional consequences of sarcopenia and dynapenia in the elderly. Curr Opin Clin Nutr Metab Care 2010, 13(3):271-276.

10. Stenholm S, Harris TB, Rantanen T, Visser M, Kritchevsky SB, Ferrucci L: Sarcopenic obesity: definition, cause and consequences. Curr Opin Clin Nutr Metab Care 2008, 11(6):693-700.

11. Batsis JA, Barre LK, Mackenzie TA, Pratt SI, Lopez-Jimenez F, Bartels SJ: Variation in the prevalence of sarcopenia and sarcopenic obesity in older adults associated with different research definitions: dual-energy X-Ray absorptiometry data from the national health and nutrition examination survey 1999-2004. J Am Geriatr Soc 2013, 61(6):974-980.

12. Oh K, Lee J, Lee B, Kweon S, Lee Y, Kim Y: Plan and operation of the 4th Korea national health and nutrition examination survey (KNHANES IV). Korean J Epidemiol 2007, 29(2):139-145.

13. Baumgartner RN, Koehler KM, Gallagher D, Romero L, Heymsfield SB, Ross RR, Garry PJ, Lindeman RD: Epidemiology of sarcopenia among the elderly in New Mexico. Am J Epidemiol 1998, 147(8):755-763.

14. McDonald JH: Handbook of biological statistics. Sparky House Publishing Baltimore; 2009:141-144

15. The EuroQol Group: EuroQol-a new facility for the measurement of health-related quality of life. Health Policy 1990, 16(3):199-208

16. Kim MH, Cho YS, Uhm WS, Kim S, Bae SC: Cross-cultural adaptation and validation of the Korean version of the EQ-5D in patients with rheumatic diseases. Qual Life Res 2005, 14(5):1401-1406.

17. Nam H, Kim K, Kwon S, Koh K, Poul K: EQ-5D Korean valuation study using time trade of method. Seoul: Korea Centers for Disease Control and Prevention; 2007

18. Schrag A, Selai C, Jahanshahi M, Quinn NP: The EQ-5D—a generic quality of life measure-is a useful instrument to measure quality of life in patients with Parkinson's disease. J Neurol Neurosurg Psychiatry 2000 69(1):67-73.

19. WHO Expert Consultation: Appropriate body-mass index for Asian populations and its implications for policy and intervention strategies. Lancet 2004, 363(9403):157-163.
20. Lee B, Lee C, Lee P, Choi M, Namkoong K: Development of Korean version of alcohol use disorders identification test (AUDIT-K): Its reliability and validity. J Korean Academy of Addiction Psychiatry 2000, 4:83-92.

21. Newman AB, Kupelian V, Visser M, Simonsick E, Goodpaster B, Nevitt M, Kritchevsky SB, Tylavsky FA, Rubin SM, Harris TB: Sarcopenia: alternative definitions and associations with lower extremity function. J Am Geriatr Soc 2003, 51(11):1602-1609.

22. Park H, Park S, Shephard RJ, Aoyagi Y: Yearlong physical activity and sarcopenia in older adults: the Nakanojo Study. Eur J Appl Physiol 2010, 109(5):953-961.

23. Lee W-J, Liu L-K, Peng L-N, Lin M-H, Chen L-K: Comparisons of sarcopenia defined by IWGS and EWGSOP criteria among older people: results from the I-Lan longitudinal aging study. J Am Med Dir Assoc 2013, 14(7):528. e521-528.e527.

24. Melton L 3rd, Khosla S, Crowson CS, O'Connor MK, O'Fallon WM, Riggs BL: Epidemiology of sarcopenia. J Am Geriatr Soc 2000, 48(6):625.

25. Lim S, Kim JH, Yoon JW, Kang SM, Choi SH, Park YJ, Kim KW, Lim JY, Park KS, Jang HC: Sarcopenic obesity: prevalence and association with metabolic syndrome in the Korean Longitudinal Study on Health and Aging (KLoSHA). Diabetes Care 2010, 33(7):1652-1654

26. Size Korea: Report on the Sixth Survey of Korean anthropometry. In Korean ministry of trade, industry and energy; 2010.

27. Park $\mathrm{HS}$, Lee $\mathrm{HO}$, Sung $\mathrm{CJ}$ : Body image, eating problems and dietary intakes among female college students in urban area of Korea. Korean J Community Nutrition 1997, 2(4):505-514.

28. Cattarin JA, Thompson JK, Thomas C, Williams R: Body image, mood, and televised images of attractiveness: the role of social comparison. J Soc Clin Psychol 2000, 19(2):220-239.

29. Wardle J, Haase A, Steptoe A: Body image and weight control in young adults: international comparisons in university students from 22 countries. Int J Obes 2005, 30(4):644-651.

30. Kim BR, Han YB, Chang UJ: A study on the attitude toward control, diet behavior and food habits of college students. Korean I Community Nutrition 1997, 2(4):530-538.

31. Lee MS, Woo MK: Differences in the dietary and health-related habits and quality of diet in university students living in Daejeon. Korean I Community Nutrition 2003, 8(1):33-40.

doi:10.1186/1471-2318-14-144

Cite this article as: Kim et al:: The application of different appendicular skeletal muscle cutoff points and research definitions associated with health-related quality of life in Korean older people: data from KNHANES 2008-2011. BMC Geriatrics 2014 14:144.

\section{Submit your next manuscript to BioMed Central and take full advantage of:}

- Convenient online submission

- Thorough peer review

- No space constraints or color figure charges

- Immediate publication on acceptance

- Inclusion in PubMed, CAS, Scopus and Google Scholar

- Research which is freely available for redistribution 